\title{
Síntese de Nanotubos de Oxido de Vanádio Pelo Método Hidrotérmico Livre de Templates
}

\author{
Francisco R. Fernandes*(Graduando em Química na Universidade do Estado do Rio Grande \\ do Norte - UERN) \\ Luiz D. Souza (Prof. Adjunto do Dq e do PPGCN na Universidade do Estado do Rio Grande do \\ Norte - UERN) \\ Adriana P. B. Santos (Doutoranda em Química na Universidade Federal do Rio Grande do \\ Norte - UFRN) \\ Anne Gabriella D. Santos (Prof ${ }^{(a)}$. Dr ${ }^{(a)}$. Na Universidade do Estado do Rio Grande do Norte - \\ $U E R N)$ \\ Vinicius P. Caldeira (Prof. Dr. na Universidade do Estado do Rio Grande do Norte - UERN)
}

\begin{abstract}
Resumo: Nos últimos tempos, materiais em nanoescala tem sido fonte de estudo para vários pesquisadores, o que proporcionou grande avanço na área e uma melhor manipulação, no que diz respeito aos diversos meios de síntese e obtenção de vários tipos de nanotubos. Uma grande atenção tem sido voltada aos nanotubos de óxido vanádio (VOx-NTs), já que o óxido de vanádio tem diversas aplicações e na sua forma tubular estas são intensificadas. Contudo, nos últimos anos, os VOx-NTs tem sido sintetizados pelo método hidrotérmico alcalino por meio da utilização de templates orgânicos, que facilitam a formação da estrutura nanotubular. Neste trabalho, os VOx-NTs foram sintetizados pelo método hidrotérmico alcalino livre de templates. $\mathrm{O}$ tratamento hidrotérmico utilizado foi à temperatura de $180^{\circ} \mathrm{C}$ durante 36 horas de envelhecimento, com o objetivo de averiguar se à estas condições os VOx-NTs eram obtidos. O que foi confirmado pelos resultados das caracterizações realizadas, sendo elas DRX, MEV, TG e DSC. No DRX mostrou que os VOx-NTs obtidos se apresentavam na forma ortorrômbica, bem como que os picos de difração eram característicos da morfologia tubular. Os resultados de MEV confirmaram a morfologia nanotubular em multicamadas dosVOx-NTs. Com nanotubos com comprimentos em média de 8-20 $\mu$ m e uma média dos diâmetros externo de 0,62 até 2,2 $\mu \mathrm{m}$. As análises térmicas mostraram seus eventos de perda de massa e revelaram que depois do material obter a morfologia nanotubular houve compactação e maior estabilidade térmica dos mesmos.
\end{abstract}

Palavras-chave: Vanádio; Nanotubos; Oxido; Síntese

\section{I ntrodução}

A ciência em nanoescala têm aliciado grande atenção nos últimos anos, pela perspectiva do impacto que estas estruturas podem oferecer na melhoria da qualidade de vida e na preservação do meio ambiente (FERREIRA; RANGEL, 2009). Recentemente com os avanços na tecnologia foi possível realizar a síntese e manipulação de nanomateriais, o que gerou um grande aumento nas atividades de investigação dedicadas a nanociência e nanotecnologia (SILVA, 2012).

Estes materiais apresentam propriedades únicas e diversas aplicações potenciais. Entre as nanoestruturas os nanotubos têm atraído grande interesse desde a identificação de Iijima, 
(1991) de nanotubos de carbono, já pela particularidade de sua forma tubular, que permite acesso a três diferentes regiões de contato, superfície interna, externa e suas extremidades (YAN et al., 2008).

Desde da descoberta dos nanotubos de carbono em 1991, um grande empenho tem sido depositado nas sínteses de diversos tipos de nanotubos. Os nanotubos de óxido de vanádio (VOx-NTs) são de grande interesse já que óxidos de vanádio são largamente aplicados em catálise e em eletroquímica (LI et al., 2006). Na forma tubular o óxido de vanádio tem suas aplicações intensificadas pela sua estrutura, onde se nota três regiões de contato, que também fornecem canais cheios de eletrólitos para maior a condução dos íons dos locais de inserção. Hoje os nanotubos de vanádio estão sendo vastamente aplicados em eletrodos positivos e em baterias de lítio secundárias, ou bateria de íons de magnésio.

Nos métodos de síntese de nanotubos de óxido de vanádio, entre eles o mais clássico é o que utilizam templates (moldes) como rota para a formação destes materiais. Yan et al., (2008) mostrou a utilização de um nanofio de ZnO como template para síntese de nanotubos de GaN, utilizando a Deposição de Vapor Químico metalorganicos (DVQ). No qual o nanofio de ZnO é facilmente extraído através de soluções acidas ou por tratamento de redução de alta temperatura. Sínteses com a presença de templates vendo sendo amplamente utilizadas pela sua eficácia e simplicidade, como é visto em diversos trabalhos (SEDIRI et al, 2007; CHANDRAPPA et al, 2003).

Nanotubos de vanádio também podem ser obtidos sem utilização de templates, um desses métodos é pelo tratamento hidrotérmico, que foi descoberto inicialmente por Kasuga et al., (1998), que descreveram esse método de processamento químico simples e direto para a preparação de nanotubos de TiO2. A primeira síntese hidrotérmica de nanotubos de titanato a partir de uma reação de partículas de $\mathrm{TiO} 2$ e $\mathrm{NaOH}$ concentrado, acompanhado por lavagem ácida.

Os métodos fundamentados em templates são considerados simples e muito eficazes para a obtenção de nanotubos, mas há também suas desvantagens. Esta rota requer o uso de uma base, ou de um ácido ou de alta temperatura para removê-los, o que aumenta bastante o custo e o risco de fabricação em ampla escala (YAN et al., 2008), Além disso, estes moldes de estruturas constituídos de materiais orgânicos podem originar múltiplos problemas quando utilizados, como manejo de produtos tóxicos e corrosivos, contaminação de recursos hídricos e terrestres, poluição atmosférica (calcinação) e obstrução de poros do catalisador com a formação de coque em virtude da combustão incompleta durante o processo de calcinação (CALDEIRA, 2011).

O presente trabalho mostra a obtenção de VOx-NTs através de síntese hidrotérmica alcalina sem a utilização de templates. O material nanotubular foi obtido a uma temperatura de envelhecimento de $180^{\circ} \mathrm{C}$ durante $36 \mathrm{~h}$. O material obtido foi caracterizado por DRX, TG, DSC e MEV, para melhor compreensão de sua estrutura, para a determinação de sua estabilidade térmica e para observar seu rendimento e morfologia.

\section{Experimental}

Síntese hidrotérmica alcalina de Vox-NTs

Inicialmente foi feito uma solução de $10 \mathrm{M}$ de $\mathrm{NaOH}$, em seguida foi pesada determinada massa de $\mathrm{V}_{2} \mathrm{O}_{5}$ para adição na solução anteriormente preparada, que foi submetida a agitação magnética durante 25 minutos. Logo depois a mistura originada é transferida para um autoclave de teflon revestido de aço inoxidável com volume de $50 \mathrm{~mL}$, e colocado numa estufa na temperatura de $180^{\circ} \mathrm{C}$ durante $36 \mathrm{~h}$, iniciando-se o tratamento hidrotérmico. No termino da síntese os Vox-NTs são transferidos para um béquer e colocados numa estufa para secar à 100 
${ }^{\circ} \mathrm{C}$ por $4 \mathrm{~h}$. Depois de seco e resfriado o material foi lavado com álcool etílico até atingir $\mathrm{pH}$ neutro.

\section{Caracterização}

Os dados obtidos na Difração de Raios-X (DRX) do pó foi recolhido em equipamento da Rigaku modelo Mini Flex II da, usando radiação k $\alpha$ de $\mathrm{Cu}\left(\lambda=1.5406^{\circ} \mathrm{A}\right)$. As medidas foram efetivadas pelo método de varredura, de $2 \theta$ de 1 a $90^{\circ}$, passo de 0,02 gaus/s e tempo de $1 \mathrm{~s}$. As imagens de Microscopia Eletrônica de Varredura foram adquiridas através de microscópio da Tescan modelo FEG-MIRA 3 LMU. As amostras foram antes dispersas em acetona, colocadas sobre uma fita adesiva de carbono montada no porta-amostra e metalizadas com uma fina camada de Au. As curvas termogravimétricas e calorimétricas (TG/DSC) foram realizadas em equipamento da Netzsch, modelo STA 449 F3 Jupiter. Foi usado aproximadamente 5,0 mg de amostra em cadinhos de alumina que foram aquecidos até $900{ }^{\circ} \mathrm{C}$ com razão de aquecimento de $10{ }^{\circ} \mathrm{C} / \mathrm{min}$ em atmosfera inerte de $\mathrm{N} 2$.

\section{Resultados e Discussões}

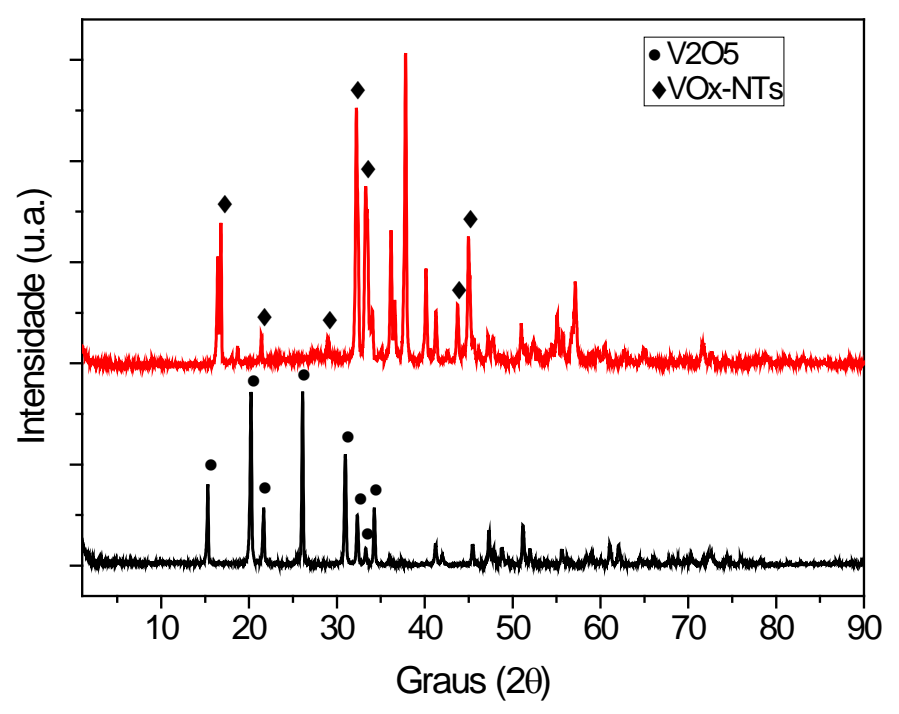

Figura 1. DRX do $\mathrm{V}_{2} \mathrm{O}_{5}$ \e dos Vox-NTs- $180^{\circ} \mathrm{C}-36 \mathrm{~h}$ 【.

Os padrões de difração do $\mathrm{V}_{2} \mathrm{O}_{5}$ e dos VOx-NTs obtidos nas sínteses são mostrados na Figura 1. Para o óxido de vanádio utilizando, como descreve Aslam et al., (2015) as reflexões intensas mostradas no gráfico indicam uma alta cristalinidade. De acordo com JCPDS-41-1426 estas reflexões correspondem a forma ortorrômbica do óxido de vanádio. As coordenadas dos picos de difração dos VOxNTs adquiridos revelam alterações quando comparadas ao óxido original, o que é referente da modificação estrutural para a forma nanotubular. E com base nos picos de reflexões obtidos por alguns pesquisadores (JIN et al, 2010; JIN et al, 2011), observase que há o aparecimento de alguns picos e o desaparecimento de outros como mostra a figura 1. Tais picos que surgem no difratograma do material VOx-NTs- $180^{\circ} \mathrm{C}-36 \mathrm{~h}$ marcados com " após ser submetido a síntese indicam com base em estudos a presença de nanotubos de vanádio puros. 


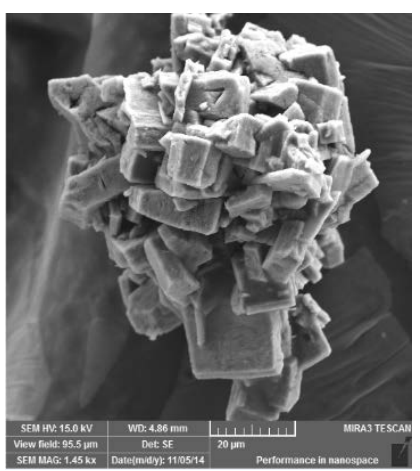

(a)

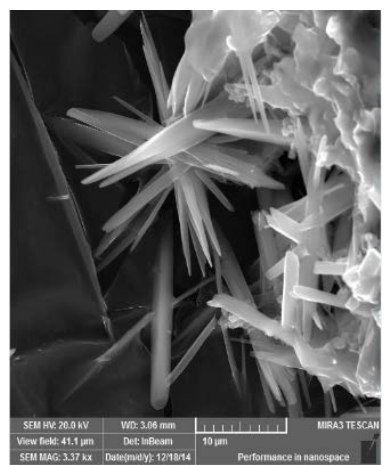

(b)

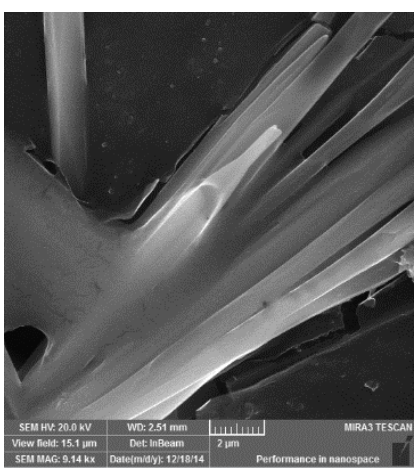

(c)

Figura 2. Imagens de MEV do $\mathrm{V}_{2} \mathrm{O}_{5}$ (a) e dos Vox-NTs- $180^{\circ} \mathrm{C}-36 \mathrm{~h}$ (b) e (c).

As imagens de MEV do óxido de vanádio original e do material obtido na síntese hidrotérmica podem ser vistos no Figura 2-(a). Pode-se verificar que a morfologia do $\mathrm{V}_{2} \mathrm{O}_{5}$ mostra uma forma de pequenos cristais compactos em uma variedade de tamanhos. Quando sujeito ao tratamento hidrotérmico alcalino, é plausível compreender que o produto final consiste sobretudo de nanotubos de óxido de vanádio, com suas extremidades abertas e superfícies lisas. O material na forma nanotubular foi obtido com um alto rendimento, contudo, essa Figura 2-(c), mostra com detalhes que o material apresenta um "oco" característico desse tipo de material. Corroborando com os resultados de raios-X dos sólidos após as sínteses. Foi notado que os VOxNTs- $180^{\circ} \mathrm{C}-36 \mathrm{~h}$ apresentam um crescimento frequentemente junto (Nefzi; Sediri, 2013; Mai et al., 2003), e que seu comprimento e diâmetro externo foram de em média 8-20 $\mu \mathrm{m}$ e $0,62-2,2 \mu \mathrm{m}$ respectivamente.

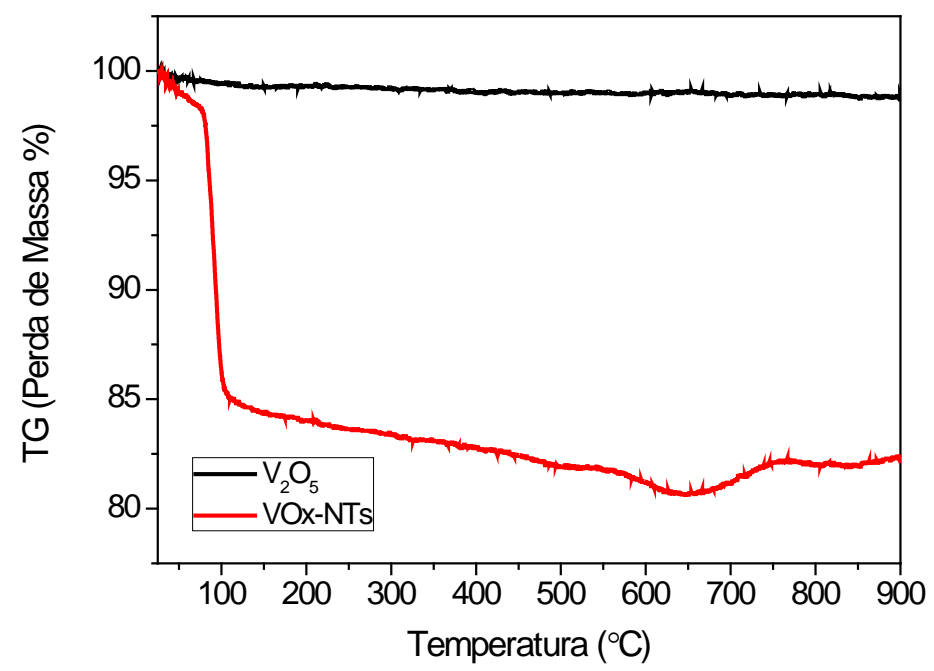

Figura 3. Curvas de TG para o Oxido de vanádio e o Vox-NTs. 


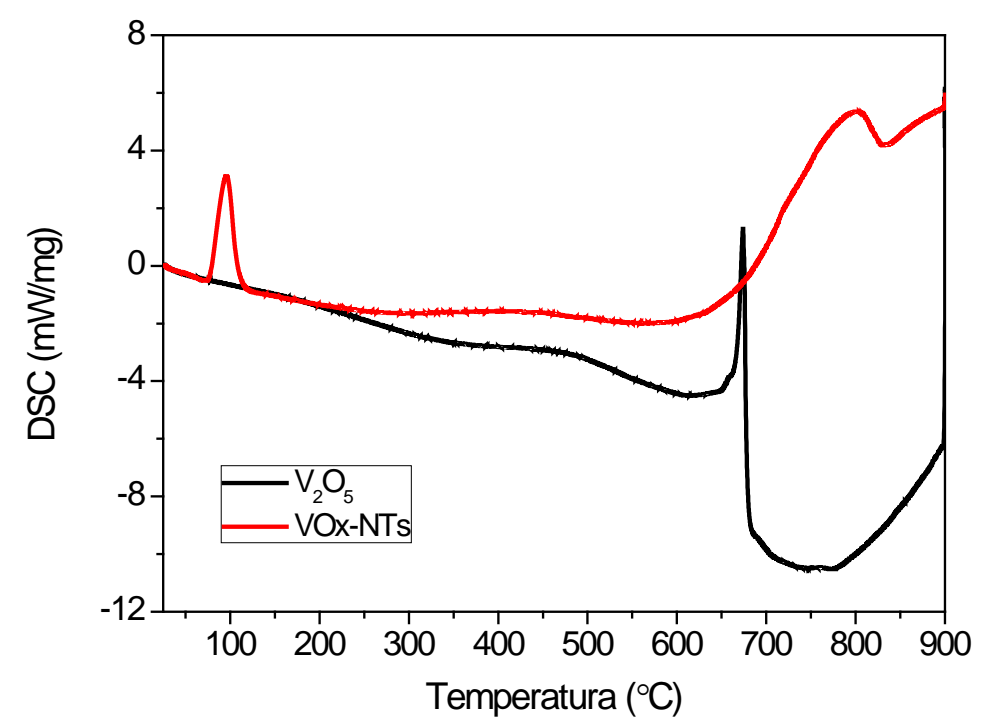

Figura 4. Curvas de DSC para o Oxido de vanádio e o Vox-NTs.

$$
\mathrm{V}_{2} \mathrm{O}_{5} \longrightarrow 2 \mathrm{VO}_{2}+1 / 2 \mathrm{O}_{2} \quad \text { Equação (1) }
$$

Os eventos térmicos para o oxido de vanádio e o VOx-NTs- $180^{\circ} \mathrm{C}-36 \mathrm{~h}$ podem ser vistos nas Figuras 3 e 4. Estas análises foram realizadas com o intuito de definir a estabilidade térmica do material. A curva de TG (Figura 3) referente ao $\mathrm{V}_{2} \mathrm{O}_{5}$, mostra a ausência de acontecimentos de perda de massa. Já a curva de TG do VOx-NTs mostra uma perda de massa inicial, por volta de $100{ }^{\circ} \mathrm{C}$, relacionada a presença de água fississorvida. A próxima ocorrência de perda de massa acontece em torno dos $400{ }^{\circ} \mathrm{C}$ e pode estar relacionado a redução de parte do $\mathrm{V}_{2} \mathrm{O}_{5}$, seguido da liberação de $\mathrm{O}_{2}$ gasoso. Esta possível redução é indicada na equação 1 (Akande et al, 2015). Há também um ganho de massa em temperaturas elevadas para o VOxNTs que pode ser atribuído a um colapso das camadas dos nanotubos pela oxidação do vanádio juntamente com a conversão para cristalitos de pentóxido da vanádio pela oxidação de $\mathrm{V}^{4+}$ para $\mathrm{V}^{5+}$ (Quites; Pastore, 2010; Chen et al., 2004).

A Figura 4 expõe as curvas de calorimetria exploratória diferencial, a curva de DSC do material sintetizado mostra um pico centralizado em $100^{\circ} \mathrm{C}$, que é devido a saída de água adsorvida no material, como mostrado antes nas curvas de TG para esta amostra. No nanotubo de oxido de vanádio ainda é observado um pico endotérmico em torno dos $465^{\circ} \mathrm{C}$, que acontece em benefício da cristalização da parte amorfa ainda no meio (Kang et al., 2014). Outro pico endotérmico referente ao VOx-NTs ocorre à $690^{\circ} \mathrm{C}$, que é devido ao seu ponto de fusão (Bentrup et al., 2003). Para a amostra com morfologia nanotubular, este pico é observado numa temperatura mais elevada, em $890^{\circ} \mathrm{C}$. Isto mostra que depois de obter a morfologia nanotubular o material se compactou, o que proporcionou uma maior estabilidade térmica.

\section{Conclusões}

Este trabalho mostrou a possibilidade de obtenção de nanotubos de vanádio, através de síntese hidrotérmica alcalina, livre de templates. Como foi mostrado no trabalho, todos os resultados revelaram que as condições de síntese foram eficazes, como a fonte de vanádio, a concentração da solução de $\mathrm{NaOH}$ e o tempo de envelhecimento, utilizados para a obtenção de VOx-NTs. Estas conclusões foram tiradas pelos resultados obtidos nos DRX e MEV. Além disso, foi visto que a morfologia nanotubular foi obtida com alto rendimento. As curvas de TG e DSC comprovaram que depois da formação dos nanotubos, o material passou a ter uma melhor 
estabilidade térmica quando comparada ao oxido de vanádio puro. Contudo, pode-se dizer que nanotubos com paredes de óxido de vanádio podem ser obtidos com alto rendimento por esta rota.

\section{Nanotube Synthesis of Vanadium Oxide By the method Hydrothermal Free Templates}

Abstract: In recent times, nanoscale materials have been the source of many researchers to study, which resulted in breakthrough area and a better handling, with respect to various modes of synthesis and obtaining various types of nanotubes. Great attention has been directed to the nanotubes vanadium oxide (VOx-NTs), since the vanadium oxide has several applications and in tubular form, these are intensified. However, in recent years, VOx-NTs have been synthesized by hydrothermal alkaline method by using organic templates that facilitate the formation of the nanotubular structure. In this work, the VOx-NTs were synthesized by alkaline hydrothermal method free templates. The hydrothermal treatment was used at a temperature of $180^{\circ} \mathrm{C}$ for 36 hours of aging, in order to ascertain whether these conditions to the VOx NTs were obtained. What has been confirmed by the results of the characterizations made, and they XRD, SEM, TG and DSC. The XRD showed that the obtained VOx-NTs are presented in the orthorhombic form as well as the characteristic diffraction peaks were the tubular morphology. SEM results confirmed the nanotubular multilayer morphology of VOx-NTs. With nanotubes with an average length of 8-20 $\mu \mathrm{m}$ and an average outside diameter of from 0.62 to $2.2 \mu \mathrm{m}$. The thermal analysis showed their mass loss events and revealed that after obtaining the nanotubular material was compression morphology and higher thermal stability thereof.

Keywords: vanadium; nanotubes; oxide; synthesis

\section{Agradecimentos}

Os autores agradecem ao apoio do CNPq, a Universidade do Estado do Rio Grande do Norte, e aos Laboratório de Catalise, Ambiente e Materiais (LACAM) e Laboratório de Análises Magnéticas e Ópticas (LAMOP).

\section{Referencias}

AKANDE, A. A. et al. Phase evolution of vanadium oxides obtained through temperature programmed calcinations of ammonium vanadate in hydrogen atmosphere and their humidity sensing properties. Materials Chemistry and Physics, v. 151, p. 206-214, 2015.

ASLAM, M. et al. Evaluation of sunlight induced structural changes and their effect on the photocatalytic activity of V2O5 for the degradation of phenols. Journal of Hazardous Materials, v. 286, p. 127-135, 2015.

BENTRUP, U. et al. Comparative study of the thermal and redox behaviour of alkali-promoted $\mathrm{V}_{2} \mathrm{O}_{5}$ catalysts. Thermochimica Acta, v. 398, p. 131-143, 2003.

CALDEIRA, Avaliação da síntese e caracterização de zeólita ZSM-5 ausente de direcionador orgânico estrutural. 2011. 88 f. Dissertação (Mestrado em Química) Universidade Federal do Rio Grandde do Norte, Natal, 2011.

CHANDRAPPA, G. T. et al. Hydrothermal synthesis of vanadium oxide nanotubes 
from V2O5 gels. Catalysis Today, v. 78, p. 85-89, 2003.

CHEN, W. et al. Synthesis of vanadium oxide nanotubes from $\mathrm{V}_{2} \mathrm{O}_{5}$ sols. Materials Letters, $v$. 58, p. 2275-2278, 2004.

FERREIRA, H. S; RANGEL, M. C. Nanotecnologia: aspectos gerais e potencial de aplicação em catálise. Química nova, Bahia, v. 32, n. 7, p. 1860-1870, 2009.

IIJIMA, S. Helical microtubules of graphitic carbon. Nature, v. 354, p. 56-58, 1991.

JIN, W. et al. Synthesis and gas sensing properties of $\mathrm{Fe}_{2} \mathrm{O}_{3}$ nanoparticles activated $\mathrm{V}_{2} \mathrm{O}_{5}$ nanotubes. Sensors and Actuators B: Chemical, v. 145, p. 211-215, 2010.

JIN, W. et al. The effect of surface morphology on the response of $\mathrm{Fe}_{2} \mathrm{O}_{3}$-loaded vanadium oxide nanotubes gas sensor. Applied Surface Science. v. 257, p. 7071, 2011.

KANG, M. et al. Conductivity, carrier density, mobility, Seebeck coefficient, and power factor in $\mathrm{V}_{2} \mathrm{O}_{5}$. Thermochimica Acta, v. 576, p. 71-74, 2014.

KASUGA, T. et al. Formation of Titanium Oxide Nanotube. Langmuir, v. 14, p. 3160-3163, 1998.

LI, H. X. et al. Factors affecting the electrochemical performance of vanadium

oxide nanotube cathode materials. Electrochemistry Communications, v. 8, p. 1693-1698, 2006.

MAI, L. et al. Cost-saving synthesis of vanadium oxide nanotubes. Solid State Communications, v. 126, p. 541-543, 2003.

NEFZI, H; SEDIRI, F. Vanadium oxide nanotubes VOx-NTs: Hydrothermal synthesis, characterization, electrical study and dielectric properties. Journal of Solid State Chemistry, v. 201, p. 237-243, 2013.

QUITES, F. J; PASTORE, H. O. Hydrothermal synthesis of nanocrystalline $\mathrm{VO}_{2}$ from poly(diallyldimethylammonium) chloride and $\mathrm{V}_{2} \mathrm{O}_{5}$. Materials Research Bulletin, v. 45, p. 892-896, 2010.

SEDIRI, F. et al. A one-step hydrothermal way for the synthesis of vanadium oxide nanotubes containing the phenylpropylamine as template obtained via non-alkoxide route. Materials Letters, v. 61, p. 1946-1950, 2007.

SILVA, E. F. B. Síntese hidrotérmica e caracterização estrutural de titanatos nanotubulares para aplicação na captura de dióxido de carbono. 2012. 119 f. Tese (Doutorado em Química) - Universidade Federal do Rio Grande do Norte, Natal, 2012.

YAN, C. et al. Tube formation in nanoscale materials. Nanoscale Res Lett, v. 3, p. 473-480, 2008. 